\title{
Anti-vascular endothelial growth factor treatment for eye diseases
}

Treatment is clearly effective but important clinical and public health implications remain

\author{
Ning Cheung assistant professor ${ }^{1}$, Dennis S C Lam honorary professor ${ }^{1}$, Tien Y Wong professor ${ }^{2}$
}

${ }^{1}$ Department of Ophthalmology and Visual Sciences, Chinese University of Hong Kong, China, Hong Kong SAR, China; ${ }^{2}$ Singapore Eye Research Institute, Singapore National Eye Centre, Singapore

\begin{abstract}
Decades of research into angiogenesis have led to a triumph in medical intervention with the development of anti-vascular endothelial growth factor (VEGF) treatment for eye diseases. Intraocular administration of anti-VEGF agents, principally ranibizumab (Lucentis) and bevacizumab (Avastin), has revolutionised the treatment of several common eye diseases that lead to blindness, including age related macular degeneration (AMD), diabetic retinopathy, and retinal vein occlusion..$^{1-3}$ The increased use of these agents in ophthalmology has enabled patients with these eye diseases to preserve or regain useful vision. Emerging evidence indicates that such treatment may account for much of the falling incidence of blindness in some developed countries over the past few years. ${ }^{4}$
\end{abstract}

The exponential rise in the use of anti-VEGF drugs for treating eye disease has far reaching implications, ${ }^{5}$ but what are the key messages for patients and their doctors? Firstly, patients should present early when their vision is affected. There is evidence that earlier treatment results in better outcomes. ${ }^{6}$ Secondly, ocular anti-VEGF treatment is not a cure, although it provides effective control of the disease by inhibiting abnormal growth and leakage of small vessels in the eye. Thus, many patients require long term repeated intraocular injections, sometimes as often as monthly, to maintain their vision. Thirdly, each injection carries a small risk of complications. Sight threatening complications are rare, but include infection (endophthalmitis), bleeding (vitreous haemorrhage), cataract, glaucoma, and retinal detachment. $^{7}$

However, three areas of uncertainty in the use of anti-VEGF agents to treat eye disease remain. Firstly, systemic safety has been a focal point of controversy. ${ }^{8}$ The intravenous administration of anti-VEGF agents in patients with cancer has a known risk of serious adverse events, including death.

Although the dose of anti-VEGF agents used for treating eye disease is minute compared with that used intravenously, there is evidence of systemic absorption and diffuse inhibition of VEGF. Clinical case series have noted antiangiogenic effects (such as regression of neovascularisation), not only in the treated eye but also in the untreated eye after bevacizumab injections. ${ }^{8}$
Moreover, case-control studies have shown marked suppression of plasma VEGF concentrations after bevacizumab injections. ${ }^{9}$ Although recent systematic reviews that include clinical trial data for ranibizumab suggest that serious adverse events are rare, ${ }^{7}$ some studies signal a possible risk of thromboembolic and non-ocular haemorrhagic events (such as stroke). ${ }^{10}$ It is unclear whether these associations are causal, given the elderly populations included in these trials and the small number of events. As for bevacizumab, its systemic safety is even more elusive because of the limited trials available and its pharmacokinetic properties (such as longer half life), which in theory may increase the risk of systemic adverse events. ${ }^{8}$

Cost effectiveness is the second area under the spotlight. At present, the cost of the two anti-VEGF agents used most commonly in ophthalmology varies widely. Ranibizumab could be as much as 40 times more expensive than bevacizumab, its off-label alternative. Cost effectiveness analysis suggests that the use of ranibizumab is not justifiable unless it is 2.5-fold more efficacious than bevacizumab. ${ }^{11}$ As the Comparison of AMD Treatment Trial indicates, this is not the case. ${ }^{1}$ Its first year results showed that ranibizumab and bevacizumab were similarly effective in treating neovascular age related macular degeneration. With around 25000 new cases of this disease diagnosed in the United Kingdom annually, replacing ranibizumab with bevacizumab as standard treatment for these cases could save close to $£ 300 \mathrm{~m}$ ( $€ 368 \mathrm{~m}$; $\$ 485 \mathrm{~m}$ ) a year. ${ }^{11}$

The third area relates to the dosing schedule of anti-VEGF treatment for eye disease. The current standard regimen, derived from landmark clinical trials of age related macular degeneration, involves monthly injections. The Comparison of AMD Treatment Trial, however, found equivalent efficacy for monthly and as needed treatment regimens of ranibizumab, with less conclusive results for bevacizumab. ${ }^{1}$ With monthly clinical assessment aided by investigative imaging (for example, optical coherence tomography), the as needed regimen almost halved the number of injections given. Such an approach is therefore an acceptable way to reduce not only the burden of cost and 
treatment but also the patient's risk of treatment related adverse events.

A new antiangiogenic agent is also on the horizon. On the basis of two phase III randomised controlled trials, the US Food and Drug Administration has recently approved the use of intraocular aflibercept injection (VEGF Trap-Eye or Eylea) for patients with neovascular age related macular degeneration. ${ }^{12}$ The first year results of these trials showed that VEGF Trap-Eye and ranibizumab injections had equivalent efficacy and safety profiles. ${ }^{12}$ Although the cost per dose (\$1850) is only slightly less than that of ranibizumab, the less frequent dosing regimen of VEGF Trap-Eye (two monthly injections after three initial monthly injections) could avoid the need for costly and time consuming monthly clinic visits for patients and their care givers.

Indeed, the therapeutic prospects of major retinal diseases have never been brighter since the advent of ocular anti-VEGF treatment. In view of the rapidly evolving clinical and economic issues, formulating evidence based treatment recommendations is not a trivial task and requires science and regulation to advance in concert. Nevertheless, the modern era of ocular anti-VEGF treatment marks an important milestone in our battle against blindness.

Competing interest: All authors have completed the ICMJE competing interests form at www.icmje.org/coi_disclosure.pdf (available on request from the corresponding author) and declare: NC and TYW had support from the National Medical Research Council (Singapore) and the
National Health and Medical Research Council (Australia) for the submitted work; TYW is a consultant for Allergan, Abbott, Bayer, Novartis, and Pfizer; no other relationships or activities that could appear to have influenced the submitted work.

Provenance: Not commissioned; externally peer reviewed.

1 Rosenfeld PJ. Bevacizumab versus ranibizumab for AMD. N Engl J Med 2011;364:1966-7.

2 Cheung N, Mitchell P, Wong TY. Diabetic retinopathy. Lancet 2010;376:124-36.

3 Wong TY, Scott IU. Clinical practice. Retinal-vein occlusion. N Engl J Med 2010;363:2135-44.

4 Cheung N, Wong TY. Changing trends of blindness: the initial harvest from translational public health and clinical research in ophthalmology. Am J Ophthalmol 2012;153:193-5.

5 Keenan TD, Wotton CJ, Goldacre MJ. Trends over time and geographical variation in rates of intravitreal injections in England. Br J Ophthalmol 2012;96:413-8.

6 Lim JH, Wickremasinghe SS, Xie J, Chauhan DS, Baird PN, Robman LD, et al. Delay to treatment and visual outcomes in patients treated with anti-vascular endothelial growth factor for age-related macular degeneration. Am J Ophthalmol 2012:153:678-686.e2.

7 Van der Reis MI, La Heij EC, De Jong-Hesse Y, Ringens PJ, Hendrikse F, Schouten JS. A systematic review of the adverse events of intravitreal anti-vascular endothelial growth factor injections. Retina 2011;31:1449-69.

8 Lim LS, Cheung CM, Mitchell P, Wong TY. Emerging evidence concerning systemic safety of anti-VEGF agents--should ophthalmologists be concerned? Am J Ophthalmol 2011;152:329-31.

9 Carneiro AM, Costa R, Falcao MS, Barthelmes D, Mendonça LS, Fonseca SL, et al. Vascular endothelial growth factor plasma levels before and after treatment of neovascular age-related macular degeneration with bevacizumab or ranibizumab. Acta Ophthalmol 2012;90:e25-30.

10 Schmucker C, Loke YK, Ehlken C, Agostini HT, Hansen LL, Antes G, et al. Intravitreal bevacizumab (Avastin) versus ranibizumab (Lucentis) for the treatment of age-related macular degeneration: a safety review. Br J Ophthalmol 2011:95:308-17.

11 Raftery J, Clegg A, Jones J, Tan SC, Lotery A. Ranibizumab (Lucentis) versus bevacizumab (Avastin): modelling cost effectiveness. Br J Ophthalmol 2007;91:1244-6.

12 Ohr M, Kaiser PK. Intravitreal aflibercept injection for neovascular (wet) age-related macular degeneration. Expert Opin Pharmacother 2012;13:585-91.

Cite this as: $B M J$ 2012;344:e2970

(c) BMJ Publishing Group Ltd 2012 